\title{
X-ray Diffraction Studies of Multiple Orientation in Poly(9,9-bis(2-ethylhexyl)fluorene-2,7-diyl) Thin Films
}

\author{
Matti Knaapila, ${ }^{*}, \dagger$ Benjamin P. Lyons, ${ }^{\ddagger}$ Kaisa Kisko, ${ }^{\dagger}$ Joel P. Foreman, ${ }^{\ddagger}$ Ulla Vainio, ${ }^{\dagger}$ \\ Milena Mihaylova,${ }^{\S}$ Oliver H. Seeck, ${ }^{\S}$ Lars-Olof Pålsson, ${ }^{\ddagger}$ Ritva Serimaa, ${ }^{\dagger}$ \\ Mika Torkkeli, ${ }^{* \dagger} \dagger$ and Andrew P. Monkman*,
}

Division of X-ray Physics, Department of Physical Sciences, University of Helsinki, P.O. Box 64, Helsinki FIN-00014, Finland, Department of Physics, University of Durham, South Road, Durham DH1 3LE, United Kingdom, and Institut für Festkörperforschung, Forschungszentrum Jülich GmbH, D-52452 Jülich, Germany

Received: May 8, 2003; In Final Form: September 1, 2003

\begin{abstract}
The structural investigation of poly(9,9-bis(2-ethylhexyl)fluorene-2,7-diyl) (PF2/6) in aligned thin films is presented. Formation of a thickness dependent triaxial texturing is identified in thermotropically aligned films. X-ray reflectivity measurements reveal good macroscopic quality, and polarized photoluminescence and dichroic ratios in absorption indicate clear axial alignment. Grazing-incidence X-ray diffraction shows axially aligned mesomorphic structure with a distinct arrangement of helices and large correlation lengths, indicating a high local lateral order. Theoretical models produced using molecular mechanics methods suggest 5/2-helicity. The polymer chains are parallel to the substrate in the c direction. In particular, the hexagonal-like cells are flattened in the direction of the surface normal and reveal two kinds of coexistent crystallites, a multiple orientation where the greater proportion of the crystallites have one crystal axis a perpendicular to the substrate surface, whereas a smaller proportion is aligned with the crystal axis a parallel to the surface. In thinner films the former class of orientation is usually dominant, while the proportion of the parallel orientation type increases with prolonged annealing.
\end{abstract}

\section{Introduction}

Design of electro-optical properties of $\pi$-conjugated polymers requires detailed understanding of the structure ${ }^{1}$ and the structure-property relationships, ${ }^{2}$ especially if aligned thin films are to be used. In general, self-assembly in hairy-rodlike polymers, such as poly(alkylthiophenes) (PATs), ${ }^{3}$ leads to highly ordered molecular packing with enhanced electronic characteristics. ${ }^{4}$ Alkyl-substituted polyfluorenes (PFs), which have excellent electro-optical properties, ${ }^{5}$ reveal complex interplay between emissive properties and intra- and intermolecular structure and defects, ${ }^{6}$ long-lived electrophosphorescence and delayed electroluminescence at low temperature, ${ }^{7}$ and complex energy transfer processes, when doped. ${ }^{8,9}$ Also, PFs exhibit thermotropic liquid-crystallinity (LC), leading to directed self-assembly when annealed on rubbed substrates. This results in emission of linearly polarized light, when used in polarizing light-emitting diodes (LEDs), ${ }^{10}$ and enhances mobility when utilized in thinfilm transistors (TFTs) ${ }^{11}$ However, the self-assembled microstructure of this class of materials in aligned thin films is not well understood. The structures of two widely used PFs, poly(9,9-(di- $n, n$-octyl)fluorene) (PF8) ${ }^{12,13}$ and poly(9,9-bis(2-ethylhexyl)fluorene-2,7-diyl) (PF2/6), ${ }^{14}$ have been studied only in aligned fibers and isotropic films ${ }^{15-17}$ and in axially aligned

* Corresponding authors. Matti Knaapila, Telephone: +358-9-19150627. Fax: +358-9-191-50639. E-mail: matti.knaapila@helsinki.fi. Mika Torkkeli, Telephone: +358-9-191-50643. Fax: +358-9-191-50639. E-mail: mika.torkkeli@helsinki.fi. Andrew P. Monkman, Telephone: +44-191-334-3616. Fax:+44-191-334-3585. E-mail: a.p.monkman@ durham.ac.uk.

$\dagger$ University of Helsinki.

$\doteqdot$ University of Durham

$\S$ Institut für Festkörperforschung. films ${ }^{14}$ (only measured in the normal direction) with electron diffraction. Detailed X-ray diffraction studies of large area films oriented on rubbed substrates have hitherto remained a challenge. ${ }^{16}$

The first detailed structural investigation of PF2/6 in aligned thin films is presented here. X-ray reflectivity measurements reveal good macroscopic quality, and polarized PL and absorption dichroic ratios in absorption indicate clear axial alignment. Grazing-incidence X-ray diffraction (GIXD) shows an aligned mesomorphic structure with a distinct arrangement of helices and large correlation lengths indicating high local lateral order. Theoretical models produced using molecular mechanics methods confirm this. The polymer chains are parallel to the substrate in the $\mathbf{c}$ direction. The cells are flattened in the direction of the surface normal and, in particular, reveal a multiple orientation where the greater proportion of the crystallites have the crystal axis a perpendicular to the substrate surface but a small proportion are aligned with the crystal axis a parallel to surface. This triaxial texture is thickness dependent: In thinner films which are somewhat better aligned, the former class of orientation is usually dominant, while the proportion of the parallel orientation type increases with prolonged annealing. These results reveal the complex structural behavior of PF2/6 in aligned thin films and shed new light on the optimization of liquid crystalline conjugated polymers.

\section{Experimental and Computational Methods}

2.1. Materials. The preparation of $P F 2 / 6^{18}$ and an oriented polyimide (PI) layer ${ }^{19}$ are described elsewhere. PF2/6 was dissolved in toluene and spin-coated onto a pretreated PI 
substrate at $2500 \mathrm{rpm}$ for $60 \mathrm{~s}$, subsequently annealed at 175 ${ }^{\circ} \mathrm{C}$, and cooled at a rate of $3{ }^{\circ} \mathrm{C} / \mathrm{min}$. The molecular axis (denoted as the $\mathbf{c}$ axis) is expected to align along the surface in the rubbing direction. Case I was prepared from a $10 \mathrm{mg} / \mathrm{mL}$ solution, and case II, from a $2 \mathrm{mg} / \mathrm{mL}$ solution. Both were annealed in a vacuum for $2 \mathrm{~h}$. Cases III and IV were prepared from $2 \mathrm{mg} / \mathrm{mL}$ and $5 \mathrm{mg} / \mathrm{mL}$ solutions, respectively, and annealed for $3.5 \mathrm{~h}$. For X-ray reflectivity measurements some samples were coated onto glass and annealed correspondingly. Further samples were prepared from a $10 \mathrm{mg} / \mathrm{mL}$ solution but left untreated to provide an unaligned film. All samples were stored and kept in the dark or under yellow light to prevent any photo-oxidation during measurements.

2.2. Optical Spectroscopy. Polarized absorption measurements were made using a JA Woollam \& Co. VASE ellipsometer with built-in polarizers, set up in the transmission mode. A bare polyimide substrate was used as a reference. Polarized fluorescence (PL) measurements were made in a Jobin-Yvon Fluoromax fluorimeter with Glan-Thomson polarizers inserted into the excitation and emission beams. The polarization bias of the instrument was taken into account.

The dichroic ratio for absorption is defined as $d=E_{||} / E_{\perp}$, where $E_{\|}$is the maximum value of the absorbance for light polarized parallel to the $\mathbf{c}$ axis and $E_{\perp}$ is the maximum for light polarized perpendicular to it. The dichroic ratio describes the anisotropy of the absorption process. The transition probability is maximized when the transition moment of the molecule lies parallel to the electric vector of the light.

The fluorescence anisotropy parameter $R$ was calculated according to the expression $R=\left(I_{||}-I_{\perp}\right) /\left(I_{\|}+2 I_{\perp}\right)$, where $I_{\|}$ is the maximum intensity of the emission parallel to the excitation beam and $I_{\perp}$ is the intensity perpendicular to it. This expression is equivalent to $R=\left(I_{\mathrm{VV}}-I_{\mathrm{VH}}\right) /\left(I_{\mathrm{VV}}+2 I_{\mathrm{VH}}\right)$, where the indices indicate the directions of the polarizers for excitation and emission, respectively. This expression assumes correction of any instrument bias to polarized light. Two absorption spectra were subsequently measured with the orientation direction parallel and perpendicular to the $\mathbf{c}$ axis and the polarization direction of the light. The order parameter $S$ was determined from the position of the maximum, $\lambda_{\max }$, of the parallel and perpendicular absorption curves, $A_{\|}(\lambda)$ and $A_{\perp}(\lambda)$, according to the relation $S=\left(A_{\|}-A_{\perp}\right) /\left(A_{\|}+2 A_{\perp}\right)$.

2.3. X-ray Reflection and Diffraction. $X$-ray reflectivity and GIXD measurements were performed at the W1.1 (ROEWI) beam line at HASYLAB in Hamburg (Germany). The beam was monochromatized by a double-crystal Si(111) monochromator, and the X-ray energy was $10.5 \mathrm{keV}$. The beam was focused on the sample, and the resulting beam size in the experimental hutch was $1.5 \times 4 \mathrm{~mm}^{2}$. The samples were mounted on the diffractometer by means of two translations and a horizontal tilt axis. In the measurement geometry, the $\mathbf{x}$ axis is defined normal to the surface and the $\mathbf{y}$ and $\mathbf{z}$ axes parallel to surface with $\mathbf{z}$ coinciding with the rubbing direction of the PI. The GIXD patterns were measured with the incident beam along the $\mathbf{y}$ and $\mathbf{z}$ axes, respectively. The scattering by air and the radiation damage were reduced by helium atmosphere.

In reflectivity measurements the incident angle was equal to the scattering angle. The thickness $t$ of the film has been determined from the interval in $q$ between successive minima as $t \approx 2 \pi / \Delta q$. The critical angle of total reflection is $\theta_{\mathrm{c}} \approx(1.61$ $\operatorname{mrad})[\lambda(\AA)]\left[\rho\left(\mathrm{g} / \mathrm{cm}^{3}\right)\right]^{1 / 2}$. In GIXD, the incident angle was $0.09^{\circ}$ or $0.11^{\circ}$, so selected to cut off scattering peaks due to the PI substrate. The Born approximation is valid because of a relatively large exit angle compared with the incident angle. ${ }^{20}$

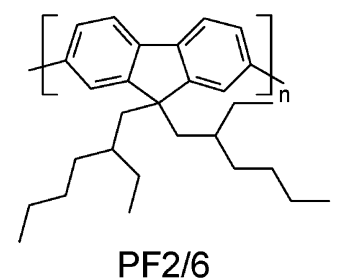

Figure 1. Chemical structure of poly(9,9-bis(2-ethylhexyl)fluorene2,7-diyl) (PF2/6).

The 2D intensity was measured with an image plate (IP) from Molecular Dynamics. Axial (in-plane and out-of-plane) scans were performed using a scintillation counter in order to calibrate the angle region and determine the lowest limits of the correlation lengths $L \approx \lambda /(\Delta 2 \theta \cos \theta)(2 \theta=$ scattering angle $)$, and for normalization, the incident flux was measured with an ionization chamber. The dimension (ver $\times$ hor) of the beam was reduced with slits to $0.1 \times 1.5 \mathrm{~mm}^{2}$ at the sample, and the distance from the sample to the receiving slits was $900 \mathrm{~mm}$. The $q$-range was $0.1-3.8 \AA^{-1}$.

The electron density map for case I was calculated using the observed diffraction intensities to derive the magnitudes of the structure factors. ${ }^{21}$ The Bragg condition may not be valid for reflections which are close to the normal direction. Therefore, these were excluded from the analysis. For the remaining reflections the Bragg condition is assumed to be valid due to the relatively large angular spread in the axial orientation in comparison with the Bragg angle $\theta<8^{\circ}$. The $10 / 1,10 / 3,5 / 2$, and $5 / 1$ helices were tried with ideal lateral close packing, and with the phases obtained from these different models it was deduced that the $5 / 2$ helix fitted best the unit cell close packing motif.

2.4. Modeling. The model structures of a single chain of PF2/6 and the close-packed triad of PF2/6 chains were completely optimized using the conjugate gradient method with the COMPASS force field. ${ }^{22}$ Each PF2/6 oligomer is created from 10 preoptimized trans-bifluorene units so that the natural helical twist is maintained. Hence, the resulting oligomer of chain length 20 adopts a trans-trans configuration that minimizes the steric interactions between adjacent ethylhexyl side chains. Partial charges were assigned to all atoms using the charge equilibration scheme, ${ }^{23}$ and no symmetry constraints were applied. All calculations were performed with the Discover program within Materials Studio 2.2 from Accelrys Inc.

\section{Results and Discussion}

3.1. Materials. $P F 2 / 6$ (Figure 1) is a side chain mesomorphic, hole-transporting material with high photoluminescence and electroluminescence quantum yields, emitting in the blue. ${ }^{15} \mathrm{It}$ can also attain a higher degree of alignment than a related polyfluorene polymer, PF8. ${ }^{14}$ At room temperature, PF2/6 is a frozen-in LC and becomes gradually more fluid during heating while remaining birefringent. It has an order-disorder transition (ODT) to the isotropic (nonbirefringent) fluid at $\sim 170^{\circ}$. The ODT is reversible, and a large hysteresis is observed, as in PF8. ${ }^{17}$ The crystallite size improves substantially during annealing, as revealed by small-/wide-angle X-ray scattering (SAXS/WAXS). ${ }^{24}$

The material was aligned using rubbed polyimide (PI) substrates and subsequent annealing. Annealing conditions, that is, the temperature and time scale on the order of hours, were selected on the basis of the observed gradual structural changes in bulk samples. For PF8, the kinetics is faster in thin films compared with that of the bulk material ${ }^{17}$ and a more rapid process might also occur here, as suggested by the relatively 


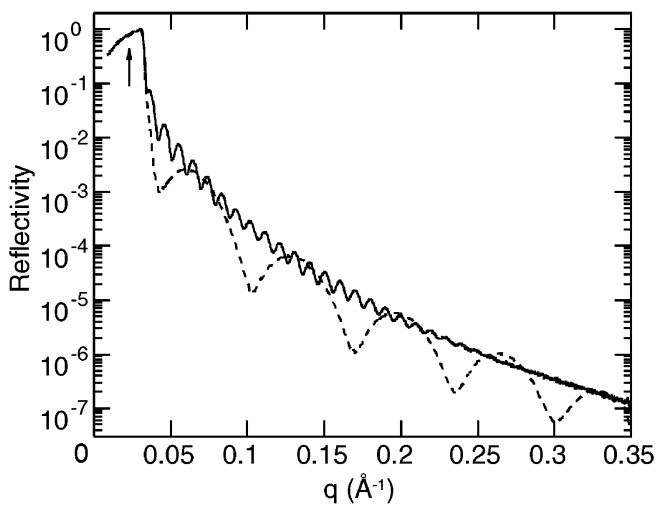

Figure 2. X-ray reflectivity curves for cases I (solid line) and II (dashed line) spun onto glass. The point marked with an arrow indicates the critical angle of PF2/6.

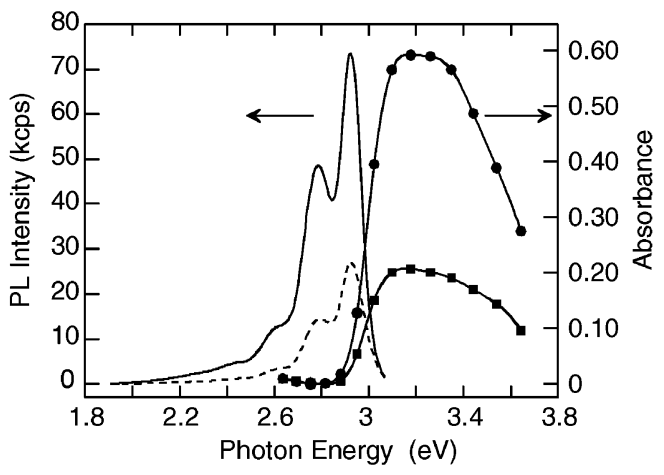

Figure 3. PL and PA spectra of case I. The excitation light $(\lambda=$ $350 \mathrm{~nm}$ ) was polarized along the $\mathbf{c}$ axis. The parallel and perpendicular polarized PL data are plotted with solid and dashed lines, respectively. The PA data were measured using polarized light with the $\mathbf{c}$ axis parallel $(\rho)$ (dots) and perpendicular $(\sigma)$ (squares) to the polarization vector of the probe light. Data were interpolated by splines.

high dichroic ratio in absorption seen after $10 \mathrm{~min}$ of annealing. ${ }^{24}$ Also other factors, such as molecular weight, ${ }^{25}$ influence the degree of alignment in PF. It is pointed out that here one of the main purposes is to compare the structural behavior to that characterized in fibers ${ }^{14}$ rather than achieve the maximum degree of alignment. For comparison, different protocols were used. In particular, case I was spin-coated from toluene solution (10 $\mathrm{mg} / \mathrm{mL}$ ) and annealed at $175^{\circ} \mathrm{C}$ for $2 \mathrm{~h}$ and cooled $3{ }^{\circ} \mathrm{C} / \mathrm{min}$, just as in previous studies. ${ }^{14}$ Cases II and III were spun from a solution of $2 \mathrm{mg} / \mathrm{mL}$, and case IV was spun from a solution of $5 \mathrm{mg} / \mathrm{mL}$. Case II was annealed similarly, but prolonged annealing (3.5 h) was used for cases III and IV.

3.2. X-ray Reflectivity. X-ray reflectivity curves for cases I and II spin-coated onto glass substrates show numerous interference fringes indicating excellent macroscopic quality and uniformity of the films; see Figure 2. Reflectivity decreases abruptly with a $q^{-4}$ slope beginning at the critical angle of the substrate, $2 \theta=0.16^{\circ}$. The interference fringes start to appear at the critical angle of PF2/6 at $2 \theta=0.11^{\circ}$, shown with an arrow. Film thicknesses are calculated to be 67 and $9 \mathrm{~nm}$, respectively, in very good agreement with direct thickness measurements. The axially aligned films show the same characteristics, but the difference in density between PI and PF2/6 (1.43 vs $\left.0.97 \mathrm{~g} / \mathrm{cm}^{3}\right)$ is rather small so that a sum of thicknesses of the layers is seen. Nevertheless, the preliminary data fitting shows quite similar behavior for the PF2/6 onto PI. ${ }^{24}$

3.3. Optical Spectroscopy. Figure 3 shows the polarized fluorescence (PL) and absorption (PA) spectra for case I. The polarized PL for case II is presented in Figure 4. The dichroic

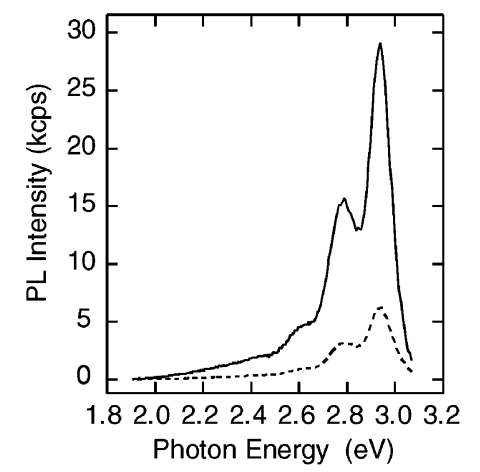

Figure 4. PL of case II. The excitation light $(\lambda=350 \mathrm{~nm})$ was polarized along the $\mathbf{c}$ axis. The solid and dashed lines show parallel and perpendicular polarized PL data, respectively.

ratios are 2.86 and 2.15 , the absorption order parameters are 0.38 and 0.28 , and the fluorescence anisotropies are 0.43 and 0.56 for cases I and II, respectively, being direct evidences of overall alignment. The alignment, not only the crystallite size, is improved by the longer annealing, resulting in corresponding values of $3.79,0.48$, and 0.62 , respectively, for case III. Unaligned films exhibited an order parameter of $\sim 0$ in absorbance, in conjunction with a very low fluorescence anisotropy of $\sim 0.01$, indicating isotropic orientation in the plane of the surface. Good in-plane orientation has been previously shown. ${ }^{15}$ When calculating the absorption dichroism for the $9 \mathrm{~nm}$ thick films, substrate scattering was found to introduce rather large errors, accounting for the low value of dichroic ratio estimated for the case II films. The slightly smaller anisotropy in fluorescence relative to absorption in Figure 3 and the very low fluorescence anisotropy of the isotropic samples are caused by inter- and intrachain excitation energy transfer to lower energy conjugation segments, whose emission dipole moments lie in different directions relative to those of the initially excited segments. The alignment of the chains leads to the alignment of the dipole moments, so the energy transfer can only partially depolarize the emission and anisotropy is mostly retained. In the isotropic case, rapid exciton migration between the random distribution of dipole moments depolarizes the emission completely. Case I shows peaks at 420 and $442 \mathrm{~nm}$ before and 424 and $445 \mathrm{~nm}$ after annealing. This red shift is of the same order for cases II-IV, reflecting an increasing average effective conjugation length due to the better local ordering of the backbones which is also seen in SAXS/WAXS measurements. ${ }^{24}$ However, the red shift is small, indicating that the intermolecular interactions are not much changed on the significant increase of the crystallite size in annealing.

3.4. X-ray Diffraction and Modeling. 3.4.1. Multiple Orientation. GIXD patterns in Figure 5 display hexagonal-like packing of the highly rodlike chains and high axial and in-plane orientation. Although the samples are the same as those described in Figures 3 and 4, the measurements were not made in situ, and therefore, the degree of alignment derived from the angular spreads of the reflections is not a directly comparable quantity. The structure resembles that observed in aligned fibers, ${ }^{14}$ where it is characterized by a trigonal unit cell containing three $5 / 1$ or $5 / 2$ helical chains. Also, the relative GIXD intensities of the first $h k 0$ reflections match the powder $\mathrm{X}$-ray diffraction pattern of the isotropic bulk material measured by SAXS/WAXS. ${ }^{24}$

However, Figure 5a actually shows two hexagonal patterns, one with a hexagonal axis a normal to the surface (cf. black dashed lines in Figure 5a) and another where a hexagonal axis a lies in the surface plane (cf. white dashed lines in Figure 5a). 


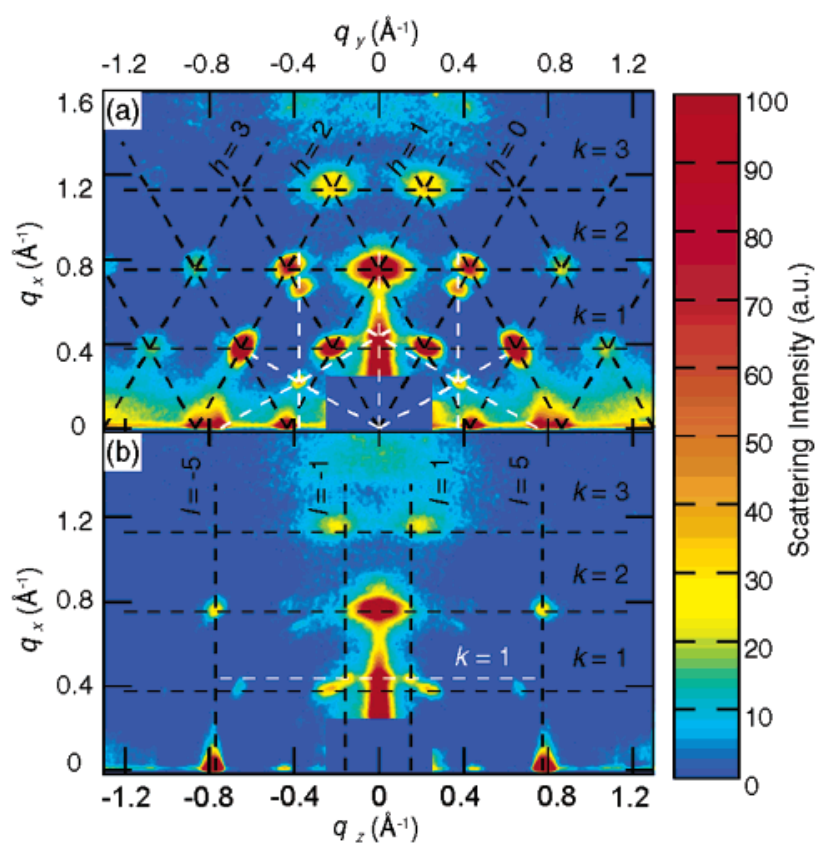

Figure 5. GIXD patterns of case I. (a) Incident beam along the $\mathbf{z}$ axis (b) Incident beam along the $\mathbf{y}$ axis. The black dashed lines show primary reflection planes of orientation type I, and white dashed lines show those of type II.

In both cases, the $\mathbf{c}$ axis is also in the surface plane. The intensity of the first pattern is 15 times higher. Since this ratio varies from one case to the other and because the observed peaks cannot fit any other single structure, these patterns clearly arise from two distinct orientation types of a hexagonal structure, denoted as types I and II, respectively. Type I is the majority class here, and type II shows only a few-but very clearreflections due to the relatively weak intensity. The positions and relative intensities are very closely the same for both types, where corresponding reflections can be observed. To our knowledge, this is the first observation of multiple orientation, triaxial texture for PF chains in axially aligned thin films. To date, stretch aligned poly ( $p$-phenylenevinylene) has been found to adopt either (200) or (110) planes oriented parallel to the surface. ${ }^{26}$ For stretch aligned solution cast PAT monoaxial distributions, "inverse combs" 27 have been reported. In thin films, depending on the processing conditions, the in-plane oriented PAT lamellae can show two different orientationseither parallel or normal to the substrate. ${ }^{4,28}$ Furthermore, it is argued that the two observed orientations also represent two slightly different crystalline forms of PF2/6.

The degree of axial orientation may be estimated by comparing the relative magnitudes of $h k 0$ reflections which are barely visible in Figure 5b to those observed in Figure 5a. From, for example, the 120 reflection, this ratio is about 20. Clearly, this suggests a higher axial orientation than that from the basis of the photoabsorption data in Figure 3. One reason may be that, there, each PF2/6 polymer, whether amorphous or crystalline, contributes to photoabsorption, while X-ray diffraction is sensitive to the aligned crystalline part only.

3.4.2. Thin Film Structure of the Two Orientation Types. The indexation of the reflections in Figure 5 is collected in Table 1. The unit cell parameters $a$ and $b$ correspond on average to the hexagonal lattice constant $a$ determined in fibers. ${ }^{14}$ The slight flattening of the unit cell normal to the surface, $\sim 1.4 \%$, is expected to result from stress. Therefore, we continue to refer to the structure as hexagonal, though orthogonal or pseudohexagonal might be a more appropriate phrase. For example, the
TABLE 1: Main GIXD Reflections in Figure 5: Positions, Corresponding Bragg Distances, $d$, Correlation Lengths, $L$, and the Indexation for the Orientation Types I and II

\begin{tabular}{|c|c|c|c|c|c|c|}
\hline$q_{x}\left(\AA^{-1}\right)$ & $q_{y}\left(\AA^{-1}\right)$ & $q\left(\AA^{-1}\right)$ & $d(\AA)$ & $L(\AA)$ & $h k l$ type I & $h k l$ type II \\
\hline 0.012 & -0.865 & 0.865 & 7.26 & & $0-20$ & \\
\hline 0.013 & -0.752 & 0.752 & 8.35 & & & -210 \\
\hline 0.013 & -0.436 & 0.436 & 14.40 & & $0-10$ & \\
\hline 0.013 & 0.436 & 0.436 & 14.40 & 230 & 010 & \\
\hline 0.012 & 0.749 & 0.749 & 8.39 & 270 & & $2-10$ \\
\hline 0.013 & 0.860 & 0.860 & 7.31 & 190 & 020 & \\
\hline 0.219 & -0.375 & 0.434 & 14.46 & & & -110 \\
\hline 0.218 & 0.378 & 0.436 & 14.41 & & & 100 \\
\hline 0.456 & -1.479 & 1.547 & 4.06 & & & -430 \\
\hline 0.381 & -1.073 & 1.139 & 5.52 & & $1-30$ & \\
\hline 0.383 & -0.645 & 0.750 & 8.38 & & $1-20$ & \\
\hline 0.381 & -0.217 & 0.438 & 14.33 & & $1-10$ & \\
\hline 0.381 & 0.217 & 0.438 & 14.34 & 270 & 100 & \\
\hline 0.381 & 0.645 & 0.749 & 8.39 & & 110 & \\
\hline 0.387 & 1.079 & 1.146 & 5.48 & & 120 & \\
\hline 0.437 & 0.001 & 0.437 & 14.37 & & & 010 \\
\hline 0.661 & -0.369 & 0.757 & 8.30 & & & -120 \\
\hline 0.663 & 0.368 & 0.758 & 8.28 & & & 110 \\
\hline 0.764 & -1.294 & 1.503 & 4.18 & & $2-40$ & \\
\hline 0.748 & -0.872 & 1.149 & 5.47 & & $2-30$ & \\
\hline 0.755 & -0.423 & 0.865 & 7.26 & & $2-20$ & \\
\hline 0.789 & -0.433 & 0.900 & 6.98 & & $2-21$ & \\
\hline 0.758 & 0.002 & 0.758 & 8.29 & 210 & $2-10$ & \\
\hline 0.752 & 0.421 & 0.862 & 7.29 & & 200 & \\
\hline 0.789 & 0.434 & 0.901 & 6.97 & & 201 & \\
\hline 0.766 & 0.868 & 1.158 & 5.43 & & 210 & \\
\hline 0.778 & 1.244 & 1.467 & 4.28 & & 220 & \\
\hline 1.150 & -1.061 & 1.565 & 4.01 & & $3-40$ & \\
\hline 1.160 & -0.222 & 1.181 & 5.32 & & $3-20$ & \\
\hline 1.158 & 0.224 & 1.180 & 5.33 & & $3-10$ & \\
\hline 1.138 & 1.064 & 1.558 & 4.03 & & 310 & \\
\hline 1.541 & -0.375 & 1.586 & 3.96 & & $4-30$ & \\
\hline 1.529 & 0.363 & 1.572 & 4.00 & & $4-10$ & \\
\hline 0.735 & -0.746 & 1.047 & 6.00 & & $2-1-5$ & \\
\hline 0.739 & 0.731 & 1.040 & 6.04 & & $2-15$ & \\
\hline 0.020 & -0.773 & 0.773 & 8.13 & 220 & $00-5$ & $00-5$ \\
\hline 0.017 & -0.432 & 0.433 & 14.52 & & $0-10$ & \\
\hline 0.016 & 0.440 & 0.440 & 14.28 & 280 & 010 & \\
\hline 0.020 & 0.774 & 0.774 & 8.12 & & 005 & 005 \\
\hline 0.382 & -0.633 & 0.739 & 8.50 & & $1-20$ & \\
\hline 0.382 & -0.225 & 0.443 & 14.18 & & $1-10$ & \\
\hline 0.432 & -0.144 & 0.455 & 13.80 & & & $01-1$ \\
\hline 0.437 & 0.002 & 0.437 & 14.37 & 230 & & 010 \\
\hline 0.436 & 0.149 & 0.461 & 13.63 & & & 011 \\
\hline 0.379 & 0.255 & 0.456 & 13.77 & & 100 & \\
\hline 0.374 & 0.649 & 0.748 & 8.39 & & 110 & \\
\hline 0.760 & -0.767 & 1.080 & 5.82 & & $2-1-5$ & \\
\hline 0.758 & 0.001 & 0.758 & 8.29 & 220 & $2-10$ & \\
\hline 0.759 & 0.775 & 1.085 & 5.79 & & $2-15$ & \\
\hline 1.156 & -0.202 & 1.173 & 5.35 & & $3-20$ & \\
\hline 1.130 & 0.210 & 1.150 & 5.47 & & $3-10$ & \\
\hline 1.157 & -0.792 & 1.402 & 4.48 & & $31-5$ & \\
\hline 1.152 & 0.778 & 1.390 & 4.52 & & 315 & \\
\hline 1.355 & 0.798 & 1.572 & 4.00 & & 305 & \\
\hline
\end{tabular}

average length of the horizontal reciprocal lattice vectors observed in Figure 5a yields $d_{100}(I)=14.690 \AA=b \sin (\gamma)$ while the $q_{x}$ values indicate $a=16.53 \AA$. The structure is assumed to be a distorted form of hexagonal structure observed in fibers ${ }^{14}$ rather than a purely new architecture. Finally, note that the strain is observable, even lacking a more strict analysis of peak shifting due to geometry. ${ }^{29}$

The observed lateral packing has unusual features for helices which lack suitable symmetry of a lattice (which are $5 / q$ helices ${ }^{14}$ ). Commonly, either a triclinic unit cell with $Z=1$ or a unit cell with an equal number of left- and right-handed helices is found. ${ }^{21}$ However, in PF2/6, the suggested number of helices is three in a hexagonal or a closely hexagonal unit cell. To illustrate this packing, a density projection on the $(a b 0)$ plane 


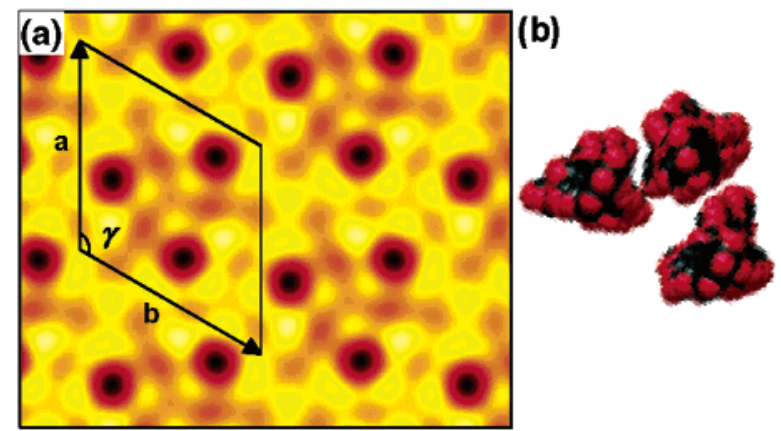

(c)

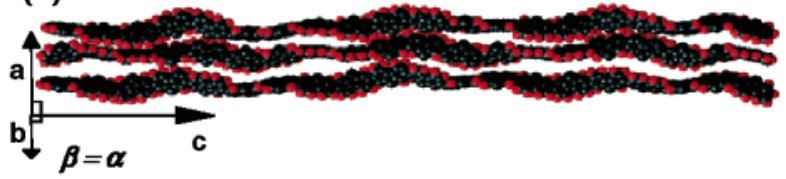

Figure 6. (a) End-on density map of orientation type I calculated from the reflection intensities for case I presented in Figure 5a. The unit cell is drawn to scale. Calculated structure of 3[PF2/6]: (b) end-on and (c) side-on. Side chains are omitted for clarity.

using Fourier synthesis ${ }^{21}$ is formed for the experimental amplitudes $\left|F_{h k 0}\right|$; see Figure 6a. It uses the observed relative structure factors for the hexagonal reflections $\left|F_{h k 0}\right|^{2}$ of type I being $30 \pm 1,100,30 \pm 1,32 \pm 4,2 \pm 2,37 \pm 8$, and $31 \pm$ 11 , for $n=h^{2}+k^{2}+h k=1,3,4,7,9,12$, and 13. The corresponding values for type II are $1.5 \pm 0.1$ and $6.7 \pm 0.5$ for $n=1$ and 3, respectively. It is stressed that the map is not unique but depends on the choice of phase angles. Nevertheless, it is concluded that the arrangement as suggested by the map is far from the "usual" packing where each chain would be surrounded by six neighbors. Instead, a more distinct motif of the three helices is indicated by the reflection intensities $\left|F_{h k 0}\right|^{2}$. Further, the map agrees more with the 5/2 helix, which has a smaller cross section diameter compared to that of the $5 / 1$ helix; see ref 14 . On the other hand, the weak $n=9$ reflection is compatible with the chain diameter of the $5 / 1$ helix because the minimum in the structure factor for a single $5 / 1$ helix is located at the position for $n=9$.

Parts $\mathrm{b}$ and $\mathrm{c}$ of Figure 6 show the molecular mechanics calculated structure of the [oligo-PF2/6] triad. The mean optimized interplanar angle between two adjacent fluorene groups of a single oligo-PF2/6 (not shown) is $144.7^{\circ}$, a value almost equal to the theoretical ideal for a $5 / 2$ helix. Earlier work $^{14}$ reported similar results, but here we have specifically included the ethylhexyl side groups. The triadic model shows an increased mean interplanar angle of $150.5^{\circ}$ and a mean interchain distance of $\sim 12.9 \AA$. Clearly, packing the chains together tends to flatten the backbone, and this likely has an effect on the electrical properties of the polymer through increased efficiency of $\pi-\pi$ transitions, that is, a red shift of the absorption band.

Along the $\mathbf{c}$ axis the observed period for the strongest reflection is $8.18 \AA$, which is also equal to that in fibers. ${ }^{14}$ Noteworthy in Figure 5b is the appearance of $l= \pm 1$ reflections near the surface normal. These belong to the type II structure. The same reflections are not observed for type I, which could be due to the scattering geometry. However, peaks with $l=$ \pm 1 should be observed at $q_{z}= \pm 0.15$ beside the -120 reflection in Figure $5 \mathrm{~b}$, which is the strongest reflection of all. It is suggested that they are present in the reported fiber diffraction patterns, ${ }^{14}$ though not mentioned. These reflections were searched for by rotating the film around the surface normal, but they were not observed. Note also that the orientation is

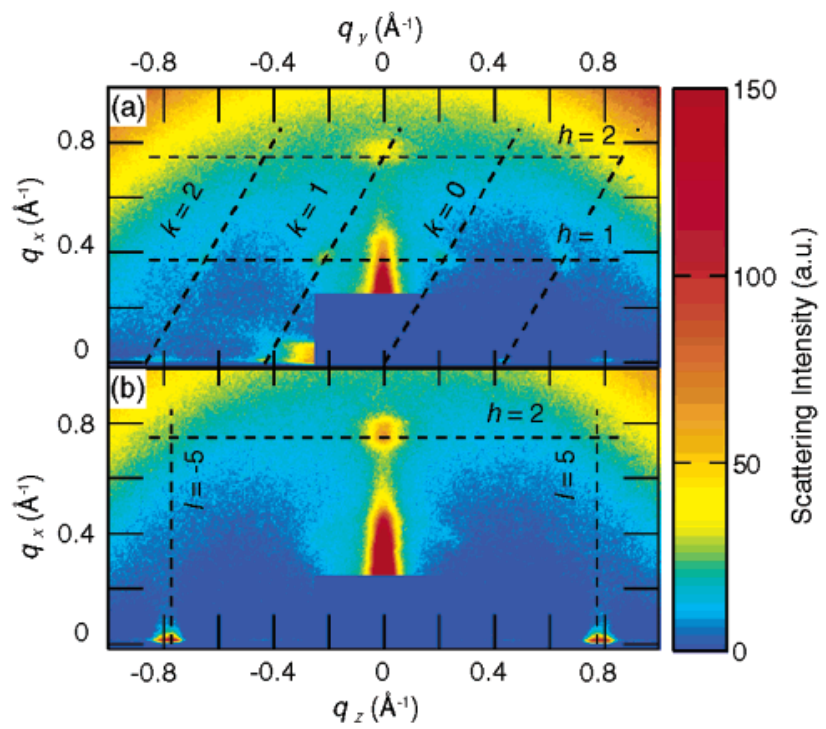

Figure 7. GIXD patterns of case II. (a) Incident beam along the $\mathbf{z}$ axis. (b) Incident beam along the $\mathbf{y}$ axis. The black dashed lines show primary reflection planes of orientation type I.

not perfect as seen by an arcing of the diffraction spots bringing scattering from the equatorial peaks into the nonequatorial layer lines. Thus, small hexagonal reflections in Figure $5 \mathrm{~b}$ can be seen, as well as, for example, the -125 reflection in Figure 5a. Therefore, the relatively strong 101 reflection (for type I) should also be visible at $q_{x}=0.38 \AA^{-1}, q_{z}=0.26 \AA^{-1}$. This indicates that type II is equivalent to the helical system observed in fibers with $c=40.8 \AA$, whereas the prevailing type I does not give reflections supporting the five monomer periodicity. Type I is then considered less ordered, which may be due to twisting of the helices around the $\mathbf{c}$ axis or a slight shift from exact five monomer periodicity in individual helices. This may be compared with, for example, the well-known crystalline transition of poly(tetrafluoroethylene) (PTFE) ${ }^{30}$ at $30^{\circ} \mathrm{C}$ due to loss of rotational order between the $15 / 7$ helices. Below the transition temperature, the PTFE shows a reflection due to the helical repeat while above the transition only reflections corresponding to monomer repeat are observed. In analogy, it is suggested that type I is the more disordered form of type II and that the different orientations are also a consequence of this difference. The less ordered chains stack on the surface in type I, as depicted in Figure 6a following organization of individual chains, whereas, with more crystalline order and stronger interpolymer interaction, the surface follows the unit cell axis, resulting in the tilted alignment. Finally, note that since only $l= \pm 5$ reflections are seen for type $\mathrm{I}$, the lattice constant $c$ is $8.18 \AA$.

The correlation lengths, $L$, (Table 1 ) are at least $250 \AA$, which is a higher value than those observed in PF8 fibers, ${ }^{12,13}$ or films, ${ }^{16}$ for instance. Due to the resolution of the grazing incidence geometry, these values represent a lower limit estimation and thus indicate large crystallite size in films. In comparison, the values of 500 and $370 \AA$ were obtained for the 100 and 010 reflections, respectively, in bulk PF2/6 annealed at $175{ }^{\circ} \mathrm{C}$ for $2 \mathrm{~h} .{ }^{24}$ These are high values for hexagonal selforganization, being comparable with those of highly ordered PATs ${ }^{31}$ where lamellar self-assembly, rather than hexagonal, facilitates the local order. Since both orientation types are LCs with slight local differences, the difference in the degree of order is not significant, which can be seen as approximately the same $L$.

Figure 7 shows the GIXD patterns of case II. Now, the material is classified predominantly as type I by its orientation. 
Since both cases I and II were prepared otherwise identically, the thinness of case II compared to case I not only promotes slightly higher fluorescence anisotropy but also promotes the type I orientation. Upon longer annealing, type II appears again and the values of 2.5 and unity for the intensity ratio between the types I and II were obtained for cases III and IV, respectively. Owing to the small number of films investigated, the results are guiding tendencies. The PI substrates may not have identical surface profiles, which may affect the crystal growth, and a simple cause of the behavior cannot be given. The multiple orientation seems not to be related to the lower degree of an axial alignment: The intensity ratio between the types increases upon longer annealing, suggesting increasing amount of type II, while the dichroic ratio increases a little, indicating better alignment along the $\mathbf{c}$ axis. The thickness dependent properties of polyfluorene are important. For instance, it has been recently shown that the chiroptical properties of a thermally annealed film of chiral substituted polyfluorene depend on film thickness. ${ }^{32}$

\section{Conclusions}

In conclusion, PF2/6 reveals a highly ordered self-assembled structure in macroscopically high quality films. When the structure is axially aligned, these films consist of two distinct orientation types from which type I has the vector a perpendicular, and type II parallel to the surface. This behavior may result from the observed packing motif of the $5 / 2$ helices and differences in crystalline order. The thinning of films enhances the degree of alignment and seems to promote crystalline type I, while longer annealing slightly improves the degree of alignment but has the opposite effect on the crystalline form, increasing the proportion of type II.

Acknowledgment. The authors thank Sony Europe Plc, EPSRC, Academy of Finland, and the Leverhulme Trust (A.P.M.). We also thank Prof. U. Scherf for the kind gift of polyfluorene.

\section{References and Notes}

(1) Winokur, M. J. Structural Studies of Conducting Polymers. In Handbook of Conducting Polymers; Skotheim, T. A., Elsenbaumer, R. L., Reynolds, J. R., Eds.; Marcel Dekker: New York, 1998; pp 707-726.

(2) Winokur, M. J.; Chunwachirasiri, W. J. Polym. Sci., B: Polym. Phys. 2003, 41, 2630-2648.

(3) Samuelsen, E. J.; Mårdalen, J. The Structure of Polythiophenes. In Handbook of Organic Conductive Molecules and Polymers; Nalwa, H. S., Ed.; Wiley: New York, 1997; Vol. 3; pp 87-120.
(4) Sirringhaus, H.; Brown, P. J.; Friend, R. H.; Nielsen, M. M.; Bechgaard, K.; Langeveld-Voss, B. M. W.; Spiering, A. J. H.; Janssen, R. A. J.; Meijer, E. W.; Herwig, P.; de Leeuw, D. M. Nature 1999, 401, 685688.

(5) Scherf, U.; List, E. J. W. Adv. Mater. 2002, 14, 477-487.

(6) Lupton, J. M.; Craig, M. R.; Meijer, E. W. Appl. Phys. Lett. 2002, 80, 4489-4491.

(7) Sinha, S.; Rothe, C.; Güntner, R.; Scherf, U.; Monkman, A. P. Phys. Rev. Lett. 2003, 90, 127402(1)-127402(4).

(8) Higgins, R. W. T.; Monkman, A. P.; Nothofer, H.-G.; Scherf, U. J. Appl. Phys. 2002, 91, 99-105.

(9) Lyons, B. P.; Wong, K. S.; Monkman, A. P. J. Chem. Phys. 2003, $118,4707-4711$.

(10) Grell, M.; Bradley, D. D. C. Adv. Mater. 1999, 11, 895-905.

(11) Sirringhaus, H.; Wilson, R. J.; Friend, R. H.; Inbasekaran, M.; Wu, W.; Woo, E. P.; Grell, M.; Bradley, D. D. C. Appl. Phys. Lett. 2000, 77 406-408

(12) Grell, M.; Bradley, D. D. C.; Ungar, G.; Hill, J.; Whitehead, K. S Macromolecules 1999, 32, 5810-5817.

(13) Grell, M.; Bradley, D. D. C.; Inbasekaran, M.; Ungar, G.; Whitehead, K. S.; Woo, E. P. Synth. Met. 2000, 111-112, 579-581.

(14) Lieser, G.; Oda, M.; Miteva, T.; Meisel, A.; Nothofer, H.-G.; Scherf, U. Macromolecules 2000, 33, 4490-4495.

(15) Tammer, M.; Higgins, R. W. T.; Monkman, A. P. J. Appl. Phys. 2002, 91, 4010-4013.

(16) Kawana, S.; Durrell, M.; Lu, J.; Macdonald, J. E.; Grell, M.; Bradley, D. D. C.; Jukes, P. C.; Jones, R. A. L.; Bennett, S. L. Polymer 2002, 43, 1907-1913.

(17) Winokur, M. J.; Slinker, J.; Huber, D. L. Phys. Rev. B 2003, 67 184106(1)-184106(11).

(18) Grell, M.; Knoll, W.; Lupo, D.; Meisel, A.; Miteva, T.; Neher, D.; Nothofer, H.-G.; Scherf, U.; Yasuda, A. Adv. Mater. 1999, 11, 671-675.

(19) Lupo, D.; Yasuda, A.; Grell, M.; Neher, D.; Miteva, T. Polyimide layer comprising functional material, device employing the polyimide layer, manufacturing the device. Eur. Pat. Appl., EP1011154, 2000, 29 pages.

(20) Dosch, H. Critical Phenomena at Surfaces and Interfaces. In Springer Tracts in Modern Physics; Hoehler, G., Ed.; Springer-Verlag: Berlin, 1992; Vol. 126.

(21) Tadokoro, H. Structure of Crystalline Polymers; John Wiley \& Sons: New York, 1979.

(22) Sun, H. J. Phys. Chem. B 1998, 102, 7338-7364

(23) Rappe, A. K.; Goddard, W. A. J. Phys. Chem. 1991, 95, 33583363.

(24) See forthcoming publications.

(25) Banach, M. J.; Friend, R. H.; Sirringhaus, H. Macromolecules 2003 , $36,2838-2844$

(26) Chen, D.; Winokur, M. J.; Masse, M. A.; Karasz, F. E. Polymer 1992, 33, 3116-3122.

(27) Prosa, T. J.; Winokur, M. J.; Moulton, J.; Smith, P.; Heeger, A. J. Macromolecules 1992, 25, 4364-4372.

(28) Aasmundtveit, K. E.; Samuelsen, E. J.; Guldstein, M.; Steinsland, C.; Flornes, O.; Fagermo, C.; Seeberg, T. M.; Pettersson, L. A. A.; Inganäs, O.; Feidenhans'1, R.; Ferrer, S. Macromolecules 2000, 33, 3120-3127.

(29) Njeh, A.; Wieder, T.; Fuess, H. Powder Diffr. 2000, 15, 211-216.

(30) Clark, E. S.; Muus, L. T. Z. Kristallogr. 1962, 117, 119-127.

(31) Prosa, T. J.; Moulton, J.; Heeger, A. J.; Winokur, M. J. Macromolecules 1999, 32, 4000-4009.

(32) Craig, M. R.; Jonkheijm, P.; Meskers, S. C. J.; Schenning, A. P. H. J.; Meijer, E. W. Adv. Mater. 2003, 15, 1435-1438. 